\title{
HYPERIMMUNOGLOBULIN D SYNDROME WITH SECONDARY AMYLOIDOSIS
}

Gustavo Roberto Lourenço ${ }^{1, \star}$, Rita de Cássia Menin ${ }^{1}$, lane Tamara Dondé ${ }^{1}$, Patrícia Milani de Moraes ${ }^{1}$, Carlos Augusto Rodrigues Padilha ${ }^{1}$, Juliana de Jesus Boscolo ${ }^{1}$, Renata Vaz de Oliveira ${ }^{1}$, Danielly Dantas Pimentel ${ }^{1}$, Maria Juliana da Silva Almeida ${ }^{1}$

1.Faculdade de Medicina de São José do Rio Preto, São José do Rio Preto (SP), Brazil.

*Corresponding author: grlourenco@icloud.com

\section{BACKGROUND}

Hyperimmunoglobulin D syndrome is a rare, autosomal-recessive genetic disorder, due to compound heterozygous or homozygous mutation in the mevalonate kinase (MVK) gene. Clinically characterized by recurrent febrile episodes typically associated with lymphadenopathy, abdominal pain, elevated serum polyclonal immunoglobulin $\mathrm{D}(\mathrm{lgD})$ level and, rarely, with secondary amyloidosis.

\section{CASE REPORT}

A 21-year-old man evaluated in January 2015 with recurrent high fever $\left(39^{\circ} \mathrm{C}\right)$ since he was 3 months old, associated with cervical and inguinal adenomegaly, and arthritis of the right foot and ankle. On physical examination, he presents ataxic gait, without other alterations. Mutations in the MVK gene were confirmed (C.803-T>C and C.77A $>C$ ) and treatment was started with methotrexate $15 \mathrm{mg}$ weekly and then associated etanercept due to treatment failure after 3 months. In March 2016, the patient evolved with recurrence of fever and adenomegaly, so we change the treatment to canakinumab $150 \mathrm{mg}$ subcutaneous each 8 weeks, evolving with reduction of inflammatory markers and resolution of the fever and adenomegaly. In 2021, he developed a new episode of fever and adenomegaly. Laboratory tests: amyloid A protein $841 \mathrm{mg} / \mathrm{dL}$. Abdominal ultrasonography: splenomegaly. Subcutaneous biopsy: search for amyloidosis by positive Congo red staining. We optimized the dose of canakinumab to $300 \mathrm{mg}$ monthly and the patient remains under clinical follow-up.

\section{CONCLUSION}

Hyperimmunoglobulin D syndrome is a genetic disease with autosomal mutation in the mevalonate kinase gene located in the long arm of chromosome 12, an essential enzyme in the functioning of the cholesterol and isoprenoid synthesis pathways. There may be a significant reduction or complete absence of their MK activity, a condition known as mevalonic aciduria. Clinical manifestations consist of fever, usually with chills, adenomegaly (mainly cervical chain, bilateral and painful), skin rash, abdominal pain, vomiting, diarrhea, arthralgia/arthritis, headache, hepatomegaly, splenomegaly, oral ulcers, and serositis. The diagnosis is defined by searching for the MVK mutation. IgD levels are usually elevated $(\geq 100 \mathrm{U} / \mathrm{mL}$ ). During febrile crises, leukocytosis and increased inflammatory markers are common. Amyloidosis is a rare but serious long-term complication, manifesting with skin bruising, enlarged muscles, heart failure, hepatomegaly and nephrotic syndrome. Diagnosed by elevated serum levels of amyloid AA and biopsy of subcutaneous fat positive to Congo red dye. Treatment consists of using Anti-TNF or Anti-IL1, which is effective to control disease activity.

\section{KEYWORDS}

Amyloidosis, Secondary amyloidosis, Hyperimmunoglobulin D syndrome. 